\title{
Palatability: from formation to possible influence on weight mass
}

\begin{abstract}
The epidemic of obesity is increasingly spreading around the globe. Many factors, some likely, others well documented both in animal both in human researches. These factors contribute to the obesity pandemic. Among these factors, there is consistent evidence that stimuli that affect the formation of organs and systems are determinants of a posteriori repercussions in the development of obesity and its comorbidities. Among these stimuli, we can mention the way in which patterns and a formation of eating habits are developed and can permeate the appearance of overweight/obesity. Nutritionally, both what we select to eat both what we ingest is associated with learning, habits and perception of taste, that is, to the palate. Does palate influence body weight gain, leading to the emergence of chronic non-communicable diseases associated with food? The aim of this mini-review is to address how food preferences and its nutritional factors can contribute to a sensory perception of food, pleasure in the consumption of certain foods and the increase food intake, can lead to overweight and obesity. The current evidences drive that palatability is an important risk factor for the development of food behavior disturbance with possible impact of overweight/ obesity on individuals.
\end{abstract}

Keywords: palatability, eating preferences, flavor, obesity
Volume 8 Issue 2 - 2018

\author{
Elizabeth do Nascimento,' Cynthia Oliveira \\ Rio Lima, ${ }^{2}$ Nathalia Cavalcanti de Morais \\ Araújo ${ }^{2}$ \\ 'Nutrition Department, Federal University of Pernambuco \\ (UFPE), Brazil \\ ${ }^{2}$ Nutrition Post graduate Program, Federal University of \\ Pernambuco (UFPE), Brazil
}

Correspondence: Elizabeth do Nascimento, Nutrition Department, Federal University of Pernambuco (UFPE), Campus Recife, PE, Brazil,Tel +55 8I 21267503, Email

nlizbeth@gmail.com

Received: January 23, 2018 | Published: March 23, 2018

\section{Introduction}

The onset of an individual's development has been extensively studied over the years. There is currently consistent evidence that in addition to the genetic component the environmental stimuli that affect the period of formation of the organs and systems are also a determinant of the repercussions a posteriori in the course of health and disease, as well as the hypothesis of the genetic component of the ingestion or preference by specific nutrients such as fat ${ }^{1}$ One of the biggest health problems in the world is the growing pandemic regarding excess body fat and its comorbidities. ${ }^{2}$ Among the predisposing factors are: polygenic inheritance, sedentary lifestyle and nutritional inadequacies. Dietary inadequacies due to deficiency or excess nutrients and/or energy in perinatal life, or at various moments in the life cycle, are strongly associated with chronic pathologies such as cardiovascular diseases, diabetes, obesity, cancers, and hypertension, others. ${ }^{2}$ In this conjunction, the way in which the food acceptance patterns and the formation of eating habits develop seem to permeate directly or indirectly the risk of the emergence of several non-transmissible diseases throughout life.

In the nutritional context, it is worth emphasizing that both the quantity and the quality of the food we eat are crucial to the repercussions in our health-disease state. What we select to eat and how much we eat are directly associated with learning, habits and perception of flavor and taste. If the taste is modifiable, therefore it can be built and deconstructed. Thus, would the taste be able to influence on body weight control, and therefore the development of chronic diseases related to palatability of food intake? Therefore, the focus of this mini review is to address how food preference and its nutritional composition may contribute to sensory perception, the pleasure of eating, increased intake, and consequently, increase of body weight. For the elaboration of this review, the following researches databases were carried out: MedLine/PubMed, SciELO, Bireme and LILACS, using the following descriptors: palatability and obesity, flavor/ taste, food and palatable intake, brain palatability, perinatal neural pathways, appetite, peptides, anorectics and orexgenes, sensory properties and organoleptic factors in studies involving both humans and animals. We selected books, review articles and originals of English and Portuguese language addressing a combination of terms in English and Portuguese without limitation of the period searched. The selection of references considers a pertinence of the objectives pursued for the scope of the study. However, only the scientific articles available on the line were selected.

\section{Discussion}

\section{Flavor, palatability and taste: definition and construction}

Flavor and/or palatability have unquestionable participation in selection and food intake. The definition of what is tasty or palatable may have a simple connection of pleasant, but a complex concept to characterize it. Determination of palatability or what is palatability involves various sensory properties (eg taste, smell, texture, temperature, visual appearance, sound and other inputs in the trigeminal nerve) and can be classified according to pleasure offered by food or how pleasant the food can be. It is evaluated by "pleasantness" or taste detector (tasting). Thus, palatability determination involves the flavor, but the classification of pleasant or flavorous is not a sufficient predictor of the consumption of a product ${ }^{3}$ and several other factors may be involved in this process. It is possible to conceptualize taste as the result of the combination of all the senses through a complex somatosensory integration of taste (flavor) and smell, touch (texture, hardness, viscosity, temperature, density, consistency, (s), view (shape, color, appearance, size, aspect, etc.) and hearing (sounds 
of crispness, softness, hardness, etc.). ${ }^{4-6}$ This sensorial set allows the perception of the most varied flavors.

\section{Factors involving food selection and / or acceptability}

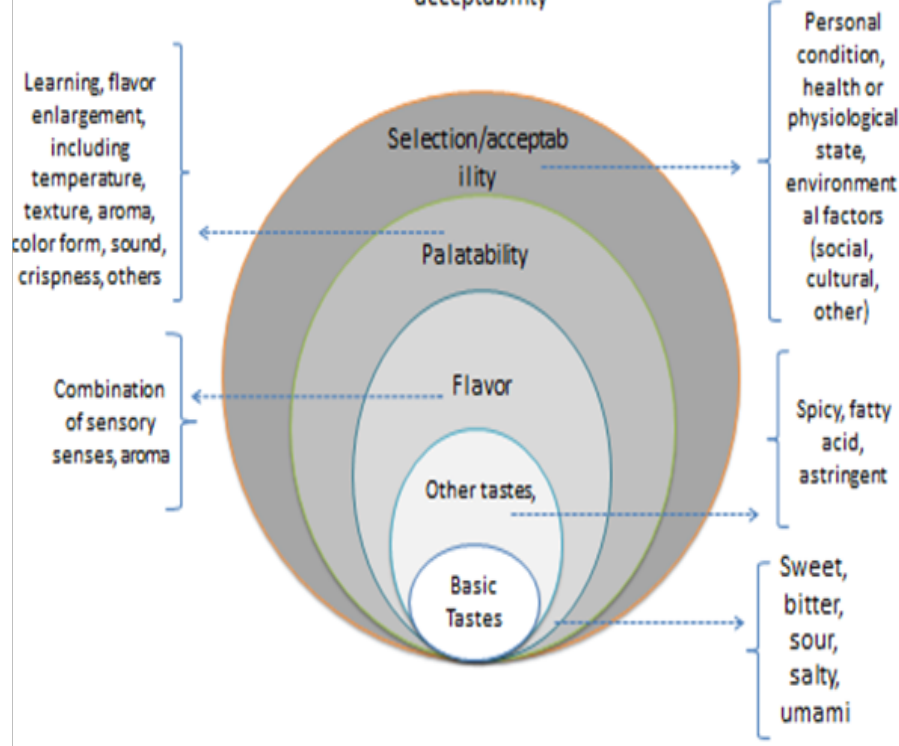

Figure I Scheme that differentiates taste, flavor and palatability.

The "palatability", in turn, associates beyond the flavor other aspects, of which we can highlight: appetite, desire, pleasure; $;, 8$ as well as the emotional and nutritional status of the individual; $;, 10$ the early childhood learning ${ }^{11,12}$ and an early exposure to food in the fetal and lactation periods. ${ }^{13}$ The taste, in turn, is limited to a sensation of the sense of the taste (or gustation) that occurs in the oral cavity of the mouth from the contact of the food on the tongue that allows the perception of the five basic tastes already defined: sweet, salty , bitter, acid ${ }^{14}$ and umami; ${ }^{6,15,16}$ Tastes are mediated by sensory receptors composed of protein structures located in cell membranes or in the cytosol (cytoplasm) that allow the interaction of signaling molecules. ${ }^{17}$ The sensory receptor has the function of transmitting to the brain information that allows the identification of the taste from the process of signal transduction and directed by the sensory nerves. ${ }^{4,18,19}$

Current evidences show a possible taste receptor for fat (fatty acids $)^{20,21}$ and, for the taste of "calcium", ${ }^{22}$ both in animals and humans, suggesting two new specific taste sensations. Animal models have provided a fundamental contribution to the historical development of understanding the basic parameters that regulate the components of our energy balance. ${ }^{3}$ However, more studies need to be carried out to better elucidate the mechanisms involved in this process ${ }^{19,23}$ and whether it occurs in humans. The possible taste of fat or specific fatty acids involves evidence in animal studies. But, although the research is not as recent the studies are still inconclusive. There is a lack of more evidences in studies performed in animals and absence of conclusive experiments in humans. ${ }^{19,24}$ Accordingly, reductions in the use of animal models, while ethically desirable, will not be feasible in the short to medium term, and indeed an expansion in activity using animal models is anticipated as the epidemic of obesity continues and spreads geographically. ${ }^{3}$ There are signs that the tongue has a specific receptor for fatty acid molecules capable of detecting fat, known as CD36. In this context, an experiment was conducted with obese individuals (with different expressions of genetic variants for CD36). The participants were submitted to the tasting of lipid emulsions with oleic acid and triolein with and without orlistat, and control emulsion (blank control). As a result the researchers observed that the presence of orlistat reduced the oral- sensory-sensitivity to triolein but not to the fatty acid suggesting the existence of a sensory component in humans for fatty acid. However, the study shows the limitation of small numbers of participants and the development of the study in a group of obese patients ${ }^{21}$ indicating the need for additional studies. The authors also suggest that the uncontrolled craving of people for certain types of hyperlipid foods (such as potato chips and fillet steak) could be genetically driven by the amount and expression of CD36 receptors and that variations in this gene could make the body more or less sensitive to taste of fat in foods. ${ }^{21}$

Studies involving genetically modified animals, lacking the CD36 gene, found that the absence of this gene prevented the detection of fat in foods by rats and that these rats had no preference for fatty foods. ${ }^{24}$ The various evidences indicate the importance of this receptor for the perception of fat taste. However, many questions remain without answers and need clarified, such as how levels of these receptors influence fat intake or body weight. ${ }^{21} \mathrm{~A}$ better understanding on action of this protein may play a relevant role in strategies to combat chronic diseases such as obesity. In Figure 1 we show in a schematic way how one can differentiate what are taste, flavor and palatability, realizing that palatability is much more complex than what can be defined by taste or flavor.

Food is of crucial importance to living beings, as it is fundamental for the survival and maintenance of homeostasis. The process of feeding is complexly determined and influenced by several mechanisms. ${ }^{14}$ According to El-Haddad ${ }^{25}$ the genetic component is a determinant that can act on taste sensitivity, indirectly on palatability, and, consequently, on food preferences. We were born with the innate ability to identify tastes with wide acceptance for sweetness and rejection for bitter and sour.${ }^{17}$ This ability is related to the intrinsic ability of survival, where we accept or select potential caloric sources and, at the same time, reject tastes that are virtually associated with toxic substances. ${ }^{19}$ In this regard, it has been advocated that the sensory properties of foods associated with higher energy content overlap to foods with low energetic value indicating that the higher energy content is sufficient to establish a preference. ${ }^{26}$

Strong evidences show that the identification of basic tastes begins in fetal life. ${ }^{27}$ The early development of the gustatory buds, innervation and development of the lingual papilla begin between the 6 th and 7 th week of fetal life, ${ }^{27}$ continuing until the 18 th week. At the 14th week of gestation the sensation of taste can already be detected. ${ }^{28}$ In humans, radiographic techniques have demonstrated that the swallowing process in fetuses is present from the 12th week of gestation ${ }^{13}$ and that swallowing of the amniotic fluid is important as a trophic factor intestinal development; and that the fetus is capable of responding to a solution of saccharose or infusion of neuropeptide $\mathrm{Y}$ (NPY) in the amniotic fluid with increasing its intake. ${ }^{21}$ However, the development of basic tastes does not all occur in the same developmental period. ${ }^{13}$ The salty taste only develops from the 4 th to the 6th month of extrauterine life and presents a peak of expression at two years of age, when the preference for this taste is higher than 
in adult life. This early attraction and development of sweet and salty tastes may in part direct appetite and choice to these tastes throughout life. ${ }^{13}$

The preferences for the various taste and formation of the palatability are constructed in early childhood with follow-up until adulthood..$^{13,29}$ Mennella et al. ${ }^{11}$ were able to demonstrate that pre and postnatal exposure to various taste and flavors increases their acceptability during the weaning and infancy periods. In the first and second trimester of gestation there is already the development of the chemosensory system capable of detecting particles dispersed in the saliva or in the retronasal air. However, it is important to remember that the respiratory process is not yet working in fetal life and that the smells along with other stimuli are relevant determinants of palatability formation. ${ }^{18}$ Taste is determined by the interaction of the dissolved particles in the saliva with the taste buds and the palatability involves beyond the gustatory perception the stimulation of the retronasal air $^{15}$ and the flavor, a set of sensorial stimuli. ${ }^{17}$ Equally, it is worth remembering that unlike innate taste, we are not born with innate preferences for smells. ${ }^{30}$ Therefore, our smell preferences consist of a learning process ${ }^{30}$ practically infinite since there is no predetermination of basic odors.

Previous experiments have shown that maternal diet during gestation and/or lactation has a profound impact on the acceptance or rejection of these same foods when babies are exposed later. ${ }^{12}$ In pregnant women, a dehydration process may occur due to frequent episodes of nausea and vomiting. ${ }^{31}$ Thus, it has been observed that there is a relationship between the increased appetite of infants and young adults due to the consumption of salty foods in mothers who presented several episodes of nausea and vomiting during pregnancy. ${ }^{31}$ A more recent study in 2012 found that children who were exclusively breastfed during the first six months of life had a preference for umami taste. ${ }^{13}$ This association is probably due to the high glutamate content of breast milk compared to that of the industrialized formulas. A wide exposure to several flavors during breastfeeding constitutes a strong and diversified learning for the child. ${ }^{12}$ This fact may have a favorable impact on the introduction and acceptance of new and varied solid foods, with a favorable effect on the formation of food preferences.

From the aforementioned factors, it can be inferred that early-life learning, maternal diet composition and genetic characteristics are determinant components of the palatability formation and of lifelong choices. ${ }^{14}$ Additional factors cannot be neglected such as maternal nutritional status or maternal diet on the food preference of their offspring. Experimental studies on rodents that have handled the lack ${ }^{14}$ or excess nutrients/energy have demonstrated a clear association with food preferences throughout life. ${ }^{32}$ Review by Portella et al. ${ }^{14}$ have demonstrated strong clinical and experimental evidence that support the fetal or early nutritional environment can significantly impact the individual's food preferences in adult life. The authors support the hypothesis that eating habits may be linked to the development of various diseases. This hypothesis stems from the fact that food preferences appear to be persistent throughout life in people who have been exposed to an adverse fetal environment. This hypothesis may, in part, explain the high risk of developing life-long metabolic diseases such as obesity, diabetes mellitus, and cardiovascular diseases. In summary, evidence from experimental and clinical studies consistently demonstrates that early life events are strongly associated with specific food preferences in adulthood, particularly for those highly palatable and energetic foods (high in carbohydrates or fat or both). The evidence that early nutritional insults promote changes in the encephalic control of food intake was observed in a study of perinatal malnutrition on the hedonic control of eating behavior. ${ }^{33}$ In this study, the authors observed that perinatal nutrition restriction promotes an increase in food intake and preference for palatable foods and that brain regions such as the amygdala and caudate putamen are implicated in this context.

In the literature, it is clear that the deficiency or excess of nutrients during the development phase ${ }^{34}$ can lead to permanent damage to body system, especially the Central Nervous System-CNS. ${ }^{35}$ In humans the development of the CNS comprises the periods of gestation, lactation and extends between 2 to 4 years of age. ${ }^{19,34}$ These periods are marked by intense proliferation and cell division and are therefore considered critical for the development of morphophysiological and biochemical processes, since any change in this period can irreversibly influence the body's metabolism and mechanisms of control. ${ }^{34}$ In this critical phase, the brain is extremely vulnerable to environmental conditions, due to growth fast and development of its organs and systems, mainly by rapid neural modifications and plastic reorganization of the CNS. Therefore, depending on the degree of deficit ${ }^{14}$ or nutritional excess and temporal extension, specific "programming" may occur to some organs or tissues permanently. 32,35

\section{Influential factors in food intake and body weight}

The relationships of palatability with various neural and peripheral circuits that culminate in the determination of food intake is complex and include several networks of sensory stimuli. Small ${ }^{15}$ argues that flavor (which is directly associated with palatability) perceived by oral sensory mechanisms is less determinant of ingestion than the sensation obtained by post-oral sensory mechanisms (distension of the stomach, circulating level of nutrients and insulin), establishing in the long term a taste preference. This means that nutritional/energy content are a better predictor of ingestion than the oral taste response, for example, the oral response to sweet taste. ${ }^{36}$ In the literature there are authors who advocate that the addition of a flavor to a balanced diet does not promote increased intake compared to a balanced diet lacking flavor or pleasant texture (44). However, the same does not proceed when the diet is high in sugar and fat ${ }^{37}$ and preference is readily formed for the flavors associated with positive post-ingestive effects. ${ }^{36}$ Thus, it can be inferred that oral sensation seems to determine acute ingestion and post-ingestion mechanisms are related to preference and consumption formation based on positive post-ingestive effects. But it is important to mention that these relationships have been studied in rodents, but in humans, little is known yet, and it is a promising area for research.

The addition of the influence of a flavor on consumption can be observed with glutamate (umami flavor). Its consumption seems to depend on the stimulation of both chemosensory perceptions (taste and post-ingestive effects) and that its sensory perception is genetically dependent. ${ }^{38}$ It is important to remember that the T1R3 gustatory cell is stimulated by both glutamate and sugar. This has led some researchers to argue that the umami flavor would be a combination of salty and sweet taste at the same time. Despite inconclusive conjectures, glutamate is a compound used to improve flavor and, therefore, palatability. Likewise, it is not negligible that palatability is a strong predictor of the quantity consumed. Palatable foods activate complex sensory systems that range from vagal stimulation to insulin secretion. 
These pathways, in turn, communicate with the reward system and are individually able to enhance the search for food and the intake of highly energetic palatable foods..$^{39}$ The increase in the consumption of highly energetic foods is a risk for the excessive gain of adipose tissue and weight and, consequently, chronic health problems.

\section{Hypothesis of cerebralcircuit dysfunction implied in the hedonic (food) system in obese individuals}

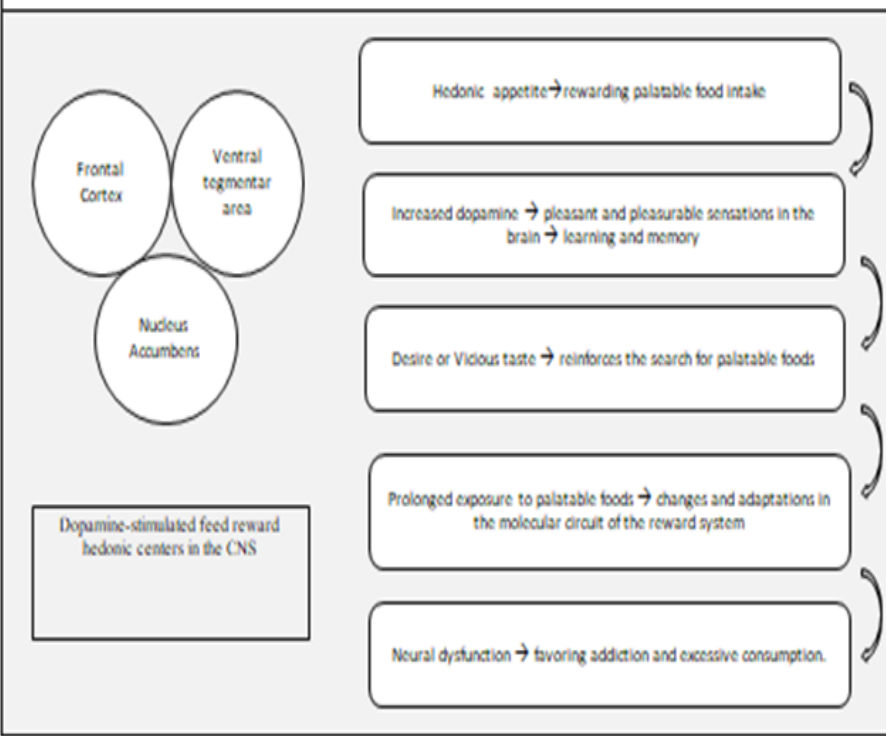

Figure 2 Hypothesis of dysfunction of the cerebral circuit involved in the hedonic or reward system (food) in obese patients.

Circuits related to food intake to satisfy homeostatic components of food and hedonic components, implies an integrated neural network of encephalic regions and orexigen peptides, anorexigenic and food reward or pleasure pathways. The reward system involves an integrated and complex neural circuit which is characterized by activations of specific brain regions and the release of neuropeptides such as: opioids, orexins and corticotropin releasing hormone, dopamine, serotonin and glutamate. ${ }^{40,41}$ It is well defined that drug use activates the reward system causing molecular adaptations throughout the brain circuit. These include changes in synaptic morphology and plasticity, as well as altered glutamatergic ${ }^{41}$ dopaminergic ${ }^{42}$ cannabinoid signaling ${ }^{43}$ and serotoninergic signaling. ${ }^{44}$ An analogue between ingestion of drug (addict) and overconsumption of palatable foods in obese individuals is based on the issue of palatable food, a daily reinforcer, which causes molecular adaptations favoring food consumption (Figure 2). ${ }^{45}$ Excessive food consumption can be harmful not only because of weight gain and its associated health risk, but also because of the adaptation that may occur in the neurobiology of the individual leading to a preference and "excessive intake" of palatable foods, far from a voluntary control and directing it to a compulsive consumption (Figure 2). ${ }^{32}$

The adaptations in the food reward system generate a positive feedback loop to improve signals that stimulate the favoring of food intake and events that block negative feedback. In addition, the data cast shows that hedonic (non-homeostatic) feed components dominate homeostatic components strongly. And the underlying plasticity of the neural circuitry that guides "overfeeding" driven by palatability is still a subject that needs to be better investigated. Figure 2 shows the hypothesis of dysfunction of the brain circuit involved in the hedonic or reward system in obese patients. Drug use stimulates the same neural circuit exemplified in the reward system of Figure 2 causing similar dependence.

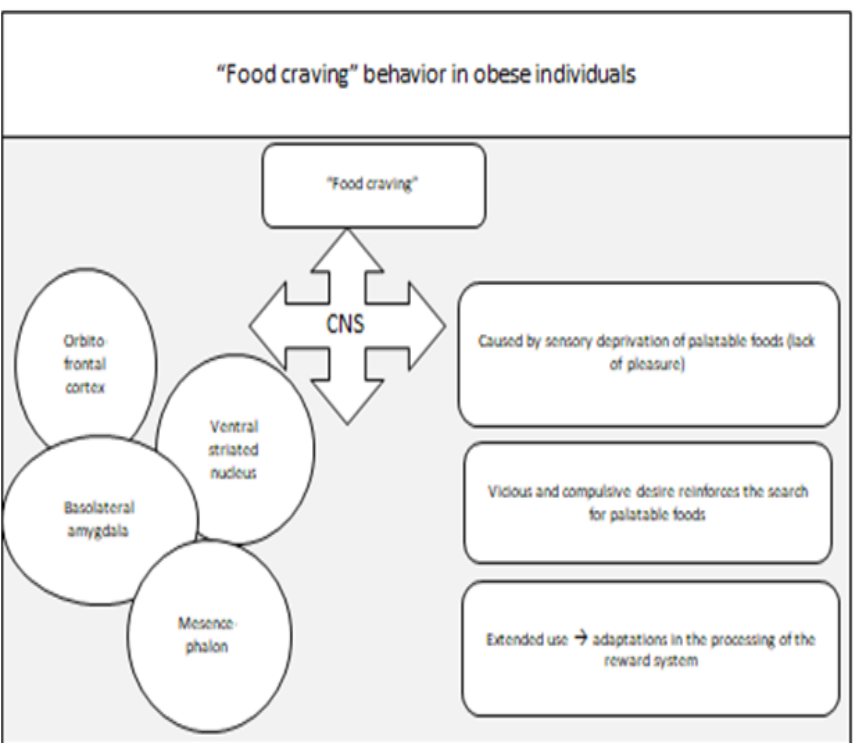

Figure 3 "Food Craving" behavior in obese individuals and the main areas of the CNS involved in this process.

The hedonic desire leads to the ingestion of palatable and rewarding foods, which stimulate the increase of dopamine levels and enjoyable and pleasurable sensations in the CNS. These sensations are learned and memorized positively leading the body to reinforce the search and desire, in a vicious way, for these palatable foods, as well as drugs. Prolonged exposure to these palatable foods leads to changes and adaptations in the processing of the food reward system, this dysfunction favors excessive food intake (overfeeding), gaining body weight and in the long run obesity. The main areas activated in the reward circuit are: nucleus accumbens, ventral tegmental area, orbitofrontal cortex and dopaminergic neurons; however, other areas of the mesolimbic system are also activated as ventral striatal nucleus, anterior cingulate cortex, ventral pallidum, amygdala, hippocampus and other specific structures of the brainstem. (adaptated from: http:// www.drugabuse.gov/publications/drugs-brains-behavior-scienceaddiction/drugs-brain.)

In animals, several methodological designs using energy-dense diets for a long time demonstrate evidence of a plasticity that may contribute to the occurrence of eating behavior as an addiction, similar to what happens in obesogenic eating behavior in humans. ${ }^{32}$ The event known as "food craving", an attractive desire to consume specific types of food (usually those with more sugar, salt and fat), is a phenomenon often experienced by most individuals. ${ }^{46}$ To a certain extent this may be positive if it serves a physiological function, ie, nutrient deficiency. But if these foods are usually high in sugar, salt and fatty, palatability rather than nutritional value appears to be the main orexigenic factor . The selection of foods craving is also associated with the culture and food preferences of individuals. ${ }^{47}$ Thus, if desirable foods are 
more energetic than the usual diet, it seems plausible that individuals with a strong attraction for palatable and/or heavily energetic foods are at high risk of gaining weight or being less susceptible to weight loss. This context would be homologous to drug addiction, where it is argued that desired drugs promote a relapse to drug use during abstinence (Figure 3). ${ }^{48}$

During the events of food craving, neural images demonstrate the activation of areas such as the orbitofrontal, caudal and medial cortex, amygdala, striatum and midbrain. ${ }^{49,50}$ In addition, scientific evidence indicates that lean and obese individuals differ in brain activity during ingestion and anticipation of palatable food intake (Figure 3). ${ }^{51}$ Obese individuals on a "diet" deprive themselves of high energy foods without, however, reducing their desire to eat these foods. ${ }^{52}$ On the other hand, dietary monotony increases the number and intensity of "craving" episodes when food deprivation is discontinued. Taken together, these reports suggest that craving is triggered by sensory deprivation (ie, the denial of food pleasure) rather than by calorie restriction (Figure 3). ${ }^{53,54}$ The manifestations are expressed as addictive behavior during access to diets high in sugar and fat and these observations closely resemble those found in humans. ${ }^{39}$

Palatable food promotes "overeating" both in satiated (satisfied) ${ }^{32}$ and in post-deprivation feedback situations when signs of hunger are triggered, emphasizing the importance of the hedonic contribution to intake irrespective of the homeostatic state..$^{55}$ The adaptations in the processing of the food reward system occur during long-term exposure to a palatable food. In these long-term adaptations or neural plasticity (study in rats with 3 weeks of exposure to sucrose) appear to occur by upregulation of markers of neuronal activity such as cFOS labeling in several limbic regions of the forebrain (including the ventral striatum, of the estrial terminal, putamen and caudate and central amygdala). ${ }^{56}$ External (environmental) stimuli are also important stimuli to overfeeding in humans, influencing choices or daily food choices. Social situation, time of day, food availability, visual stimulus, frequency and size of the meal are some plausible examples. ${ }^{57}$ Curiously, obese individuals are more responsive to environmental stimuli, and less sensitive to endogenous signs such as full satiety. In relation to visual stimulation, an interesting metaanalysis study on the correlation between neural processes and visual stimuli allows visual stimuli to be able to activate hedonic areas in the brain when the food has high energy density. ${ }^{58}$

The relationship between palatable foods and endogenous peptides associated with the pleasure cycle or hedonic eating behavior is also a field of promising interest. The palatable food modifies the activity of the opioidergic pathway (opioid peptides and their receptors), which stimulates food consumption, acquiring a prominent role in chronic overfeeding. ${ }^{55}$ In the case of animals submitted to intermittent sucrose intake, it indicates an endogenous dependence on opioids. ${ }^{32}$ The food reward system and drug or food addictions have a strong connection to the mesocorticolimbic system. In animals, the ingestion of new foods ${ }^{59}$ or palatable foods ${ }^{60}$ causes dopamine release in estritato. ${ }^{58}$ This release of dopamine in the striatum region is proportional to the pleasure that food produces and has been observed in humans from neuroimaging studies. ${ }^{15}$ However, an impaired estriate response to dopamine release (D2 receptor reduction) was observed when humans had excess body fat ${ }^{58}$ and that this attenuated response occurs when the individual gains weight, suggesting a plasticity in the human reward system as a function of metabolic or dietary changes. ${ }^{53}$

Another important group of peptides related to food intake and hedonic activation are endocannabinoids. Activation of cannabinoid type 1 (CB1) receptors occurs at various sites including hypothalamus, striatum and brainstem ${ }^{61}$ and its orexigenic effects have been associated with the inhibition of glutamatergic stimuli in the ventral striatum. ${ }^{62}$ These peptides show responses to palatable diet similar to that observed for opioids and dopaminergic pathways. Strong association also exists between leptin levels and levels of endocannabinoids since the increase of leptin in the hypothalamus reduces levels of endocannabinoids. ${ }^{63}$ Other mechanisms and intracellular signaling also participate in this complex neural network, and together the reward system and circuits traditionally associated with the hunger/ satiety cycle show functional adaptations over time in response to the palatable diet.

Palatability is undeniably a factor that drives the desire to eat (stimulates overfeeding), especially those foods that are high in sugar and fat, which stimulate hedonic areas and may be one of the risk factors that contribute to current epidemic obesity. In addition, it evokes molecular and neurobehavioral adaptations that define our long-term attitude and response to rewarding consumption, affecting the preference for macronutrients and flavors. Thus the plasticity of palatability neurobiology can infer behavioral changes including the high preference for diets high in sugar and fat or attenuated signs of satiety, and deserve more attention in research as a predisposing factor to the obesity epidemic.

Sensory stimulation, food choices and body weight. The industrialization of food has been growing on a large scale; its products are varied and are present all over the world. However, before reaching the market shelves, food is submitted to several processes, among them we can highlight the procedures of quality and food control. Industries need to test, for example, the taste, appearance and acceptability of their products and for this, use "Sensory Analysis" tests. ${ }^{64}$ According to the Institute of Food Science and Technology, ${ }^{45}$ "Sensory Analysis" can be defined as being a discipline the use several methods to trigger, measure, analyze and interpret the reactions produced by the characteristics of foods and materials as they are perceived by the senses of sight, smell, taste, touch and hearing.

Simchen et al. ${ }^{65}$ in studies with youth, adults and the elderly verified the relationship between body weight and sensory capacity. For the sensory analysis, different concentrations of each basic taste were used: the salty was represented by sodium chloride, sweet by sucrose, acid by citric acid and bitter by quinine hydrochloride. In the present study, the ability to identify and perceive odor and also the basic tastes in healthy males $(n=130)$ and women $(n=181)$, with normal weight and overweight were assessed. ${ }^{65}$ As a result, an association between overweight and reduced perception of basic tastes in adults was observed ${ }^{65}$ In another study Salbe et al. ${ }^{12}$ investigated preferences of taste and possible changes in body weight in individuals prone to obesity. The experiment consisted of 123 Indians (Pima) and 64 white volunteers, both of whom are prone to obesity. ${ }^{61}$ For the sensorial test were used solutions of skim milk ( $0.15 \%$ fat), whole milk (3.5\% fat), cream milk $(11.3 \%$ fat $)$ and cream $(37.5 \%$ fat $))$ containing a sugar content of $0 \%, 10 \%$ or $20 \% .^{12}$ The solutions were classified according to the perceived sweetness, creaminess and pleasantness (hedonic response). ${ }^{12}$ The authors found that the taste preference for highly palatable foods (rich in sugar and fat) seems to be associated with overweight and that this may favor the current obesity epidemic. ${ }^{12}$

Bartoshuk etal. ${ }^{66}$ evaluated the relationship of sweet taste and fat also with obesity. The study consisted of obese and low weight individuals 
according to body mass index, who were submitted to factorial analysis of taste in some foods and beverages and were divided into two groups: sweet food (sugar, biscuit, chocolate, candy, honey and chocolate milk) and fat foods (cheddar, mayonnaise, whipped cream, whole milk and butter). The results of this study suggest that obese people consider sweet taste less intense than non-obese individuals and that this probably intensifies sensory sensations of fat. ${ }^{66}$ The same obese group also presented preference for the group of foods with fat demonstrating a higher degree of liking. Together these preferences may contribute considerably to the high consumption of densely energetic foods and increase in body weight. ${ }^{66}$ Another study with groups of obese individuals ( $n=61$, according to the time of obesity, before or after 10 years of age) and lean $(n=31)$, aged between 20 and 45 years, evaluated the taste profiles and preference for sugar and fat. ${ }^{25}$ Sensory tests (stimuli) contained five different sweet solutions based on sucrose $(2 \%, 4 \%, 8 \%, 16 \%$, and $32 \%$ sucrose $)$ and nine different solid mixtures containing fat similar to cake coating ( $15 \%$ and $35 \%$ fat). ${ }^{25}$ The results revealed that obese individuals with body weight oscillations showed high preferences for sensory stimuli such as sweet and sweet with fat when compared to obese individuals with more stable body weight. This high sensory preference for caloric (high fat and sugar) foods in obese individuals with oscillations of weight seems to influence the vulnerability to dietary challenge rather than their cause. ${ }^{24} \mathrm{He}$ et al. ${ }^{67}$ evaluated the contribution of monosodium glutamate intake (umami taste) and overweight in humans. Humans of both sexes between 40 and 59 years old, and with use of $0.33 \mathrm{~g} /$ day of monosodium glutamate in the diet or without, showed a positive relation with BMI and prevalence of overweight. It is assumed that ingestion of oral monosodium glutamate-MSG could be affecting the regulation of appetite in the $\mathrm{CNS}$, thus contributing to the weight gain of these individuals. ${ }^{68}$

This positive relationship between ingestion of MSG and excess body weight is also consistent with data from animal studies in experimental models a few decades ago. ${ }^{44,69}$ The early injection of MSG into animals induces bilateral lesion of the ventromedial nucleus (VMH), and in the arcuate hypothalamic nucleus (ARC) from the neuronal necrosis of several regions of the CNS. ${ }^{69}$ Neonatal treatment with this experimental method that leads to the destruction of CNS cells by neurotoxicity destroys ARC and VMH cells (regions involved in food intake control), ${ }^{69}$ inducing neuroendocrine disorders and obesity. ${ }^{70}$ However, in humans this methodological study is not conclusive. ${ }^{67,71}$ Probably, its occur due to the difficulties in quantifying and controlling the oral intake of MSG, since the industries have widely used their properties (flavor enhancers) in their products. This widespread exploitation of MSG by food industries in recent decades has dramatically increased MSG consumption in the world. ${ }^{40}$ This issue raises a possible concern about the free consumption of MSG and its repercussions for the human organism in the long term, especially as this additive can contribute to a higher food intake.

Settle ${ }^{33}$ in research involving patients with diabetes mellitus evidences significant alterations in the sensitivity of the gustatory and olfactory senses in more than $60 \%$ of the patients. This fact could induce an increase in the consumption of some nutrients rich in sugar and salt, for example, in addition to their physiological needs, contributing not only to the worsening of diabetes mellitus, but also as a trigger to develop other chronic diseases such as Systemic Arterial Hypertension $(\mathrm{SAH})^{72}$ and cardiovascular diseases. ${ }^{73}$ The World Health Organization (WHO) confirms this fact and draws attention to the adverse effects of high sodium intake and increased risk for cardiovascular disease ${ }^{74-88}$ However, according Conlin, ${ }^{72}$ individual perception and sensitivity can influence the ingestion.

\section{Final considerations}

Palatability is a factor that directs food intake, especially to palatable foods densely energetic promoted mainly by the presence of sugar, sodium and fat. However, it cannot be overlooked that ingestion is directly related to the release of post-ingestion factors. The set of external and internal signals stimulate rewarding hedonic areas related to appetite and pleasure, and therefore deserve attention as possible contributing factors to the risk of development of the obesity epidemic. In this review we also highlight the early experiences in early life and inadequate maternal dietary habits (excess or scarcity) with triggers that influence the formation, acceptance and food preferences, and consequently, the quality and quantity of what is eaten, and behavior food. These factors permeate directly or the risk of lifelong metabolic disorders, such as overweight/obesity. It is also important to remember that most foods associated with high palatability are processed foods with the presence of flavor enhancers, such as monosodium glutamate, sodium itself, additives, flavors and sugars. These palatable foods are highly involved in modern life, which seeks the practicality of preparation and ease of ingestion, culminating in the reduction of the consumption of natural products by industrialized products and, consequently, in the limitation of the intake of fibers and phytochemicals with additional benefits attributed the health. In this context, it is noted that palatability seems to be a factor that directs food intake and is closely related to food choices and how much is eaten. Thus, its contribution to a high energy intake and, consequently, excessive body weight gain with risk to the emergence of obesity and related pathologies should not be neglected in combating the epidemic of obesity in the terrestrial globe.

\section{Acknowledgement}

None.

\section{Conflict of interest}

We declare no financial interest nor does any conflict of interest exist.

\section{References}

1. Chmurzynska A, Mlodzik MA. Genetics of fat intake in the determination of body mass. Nutrition Research Reviews. 2017;30 (1):106-117.

2. Rolls BJ, Roe LS, Meengs JS. The effect of increased portion size on energy intake is sustained for 11 days. Obes Res. 2005;13:A36.

3. Sørensen LB, Møller P, Flint A, et al. Effect of sensory perception of foods on appetite and food intake: a review of studies on humans. Int $J$ Obes Relat Metab Disord. 2003;27(10):1152-1166.

4. Delwiche JF. The impact of perceptual interactions on perceived flavor Food Quality and Preference. 2004;15:137-146.

5. Small DM. Flavor is in the brain. Physiol Behav. 2012;107(4):540-552.

6. Zhang Y, Proenca R, Maffei M, et al. Positional cloning of the mouse obese gene and its human homologue. Nature. 1995;374(6521):452-532.

7. Harrold. Down-regulation of cannabinoid-1 (CB-1) receptors in specific extrahypothalamic regions of rats with dietary obesity: a role for endogenous cannabinoids in driving appetite for palatable food? Brain Research. 2002;952(2):232-238. 
8. Wynne K, Stanley S, McGowan B, et al. Appetite control. J Endocrinol. 2005;184(2):291-318.

9. Rada P, Avena NM, Hoebel BG. Daily bingeing on sugar repeatedly releases dopamine in the accumbens shell. Neuroscience. 2005;134:737744.

10. Rolls BJ, Roe LS, Meengs JS. Larger portion sizes lead to sustained increase in energy intake over two days. J Am Diet Assoc. 2006;106:543549 .

11. Menella JA, Beauchamp GK. Maternal diet alters the sensory qualities of human milk and the nursling's behavior. Pediatrics. 1991;88(4):737-744.

12. Salbe AD, DelParigi A, Pratley RE, et al. Taste preferences and body weight changes in an obesity-prone population. Am J Clin Nutr. 2004;79(3):372-378.

13. Pomonis JD, Jewett DC, Kotz CM, et al. Sucrose consumption increases naloxone-induced c-Fos immunoreactivity in limbic forebrain. $\mathrm{Am} J$ Physiol Regul Integr Comp Physiol. 2000;278(3):R712-R719.

14. Skinner JD, Carruth BR, Wendy B, et al. Children's food preferences: a longitudinal analysis. J Am Diet Assoc. 2002;102(11):1638-1647.

15. Auvray M, Spence C. The multisensory perception of flavor. Conscious Cogn. 2008;17(3):1016-1031.

16. Breslin PA, Spector AC. Mammalian taste perception. Curr Biol. 2008;26;18(4):148R-155R.

17. Nicklaus S, Boggio V, Issanchou S Food choices at lunch during the third year of life: high selection of animal and starchy foods but avoidance of vegetables. Acta Pediatrika. 2005;94(7):943-951.

18. Glendinning JI, Beltran F, Benton L, et al. Taste does not determine daily intake of dilute sugar solutions in mice. Am J Physiol RegulIntegr Comp Physiol. 2010;299(5):R1333-R1341.

19. Niki M, Jyotaki M, Yoshida R, et al. Reciprocal modulation of sweet taste by leptin and endocannabinoids. Results Probl Cell Differ. 2010;52:101114.

20. Leshem M, Abutbul A, Eilon R, et al. Exercise Increases the Preference for Salt in Humans. Appetite. 1999;32(2):251-260.

21. Penna EW. Sensory methods and their applications. In: Almeida TC, editor. Advances in Sensory Analysis. São Paulo: Publishing Company Varela, 1999: p. 13-22.

22. Drewnowski A, Brunzell JD, Sande K, et al. Sweet tooth reconsidered: taste preferences in human obesity. Physiol Behav. 1985;35(4):617-622.

23. Tiggemann M, Kemps E. The phenomenology of food cravings: the role of mental imagery. Appetite. 2005;45(3):305-313.

24. Kemps E, Tiggemann M. Modality-specific imagery reduces cravings for food: an application of the elaborated intrusion theory of desire to food craving. Journal of Experimental Psychology: Applied. 2007;13(2):95104.

25. Drewnowski A, Kurth CLE, Rahaim JE, et al. Taste preferences in human obesity: environmental and familial factors. Am J Clin Nui. 1991;54(4):635-641.

26. Wansink B. From mindless eating to mindlessly eating better. Physiol Behav. 2010;100:454-463.

27. Bradley RM, Stern IB. The development of the human taste bud during the foetal period. J Anat. 1967;101(Pt 4):743-752.

28. Weingarten HP, Elston D. Food cravings in a college population. Appetite. 1991;17:167-175.

29. Michael Naim, Joseph G Brand, Morley R Kare, et al. Energy intake, weight gain and fat deposition in rats fed flavored, nutritionally controlled diets in a multichoice ("cafeteria") design. J Nutr. 1985;115:1447.

30. Rossner S. Obesity: the disease of the twenty-first century. Int J Obes. 2002;26(Suppl):S2-S4.

31. Laugarrete F. CD36 involvement in orosensory detection of dietary lipids, spontaneous fat preference, and digestive secretions. J Clin Invest. 2005;115(11):3177-3184.

32. Alsiö J. Dopamine D1 receptor gene expression decreases in the nucleus accumbens upon long-term exposure to palatable food and differs depending on diet-induced obesity phenotype in rats. Neuroscience. 2010;171(3):779-787.

33. Settle RG. The chemical senses in diabetes mellitus. In: Getchell TV, Barto Shuk, LM. Smell and taste and disease. New York, Raven Press, 1991: p. 829-843.

34. Monteleone P, Maj M. Dysfunctions of leptin, ghrelin, BDNF and endocannabinoids in eating disorders: beyond the homeostatic control of food intake. Psychoneuroendocrinology. 213;38(3):312-330.

35. El-Haddad Ma. In Utero Development of Fetal Thirst and Appetite: Potential for Programming. Reproductive Science. 2004;11(3):123-30.

36. Elinor L, Sullivan M, Susan S, et al. Perinatal Exposure to High-Fat Diet Programs Energy Balance, Metabolism and Behavior in Adulthood. Neuroendocrinology. 2012;93(1):1-8.

37. Schwartz C, Issanchou S, Nicklaus S. Developmental changes in the acceptance of the five basic tastes in the first year of life. Br J Nutr. 2009;102:1375-1385).

38. Bachmanov AA, Inoue $\mathrm{M}$, Ji H, et al. Glutamate taste and appetite in laboratory mice: physiologic and genetic analyses. Am J Clin Nutr. 2009;90(3):756S-763S.

39. Alsiö J, Olszewski PK, Levine AS, et al. Feed-forward mechanisms: Addiction-like behavioral and molecular adaptations in overeating. Front Neuroendocrinol. 2012;33(2):127-139.

40. Hermanussen M, García AP, Sunder M, et al. Obesity, voracity, and short stature: the impact of glutamate on the regulation of apetite. European Journal of Clinical Nutrition. 2006;60(1):25-31.

41. Witt M. Reutter K. Embryonic and early fetal development of human taste buds: A transmission electron microscopical study. The anatomical record; 1996.

42. Briand LA, Flagel SB, Seeman P, et al. Cocaine self-administration produces a persistent increase in dopamine D2 High receptors. Eur Neuropsychopharmacol. 2008;18(8):551-556.

43. Guyton ACE, Hall JE. Treatise on Medical Physiology. Elsevier Publishing Company; 2011.

44. Bechtholt AJ, Smith K, Gaughan S, et al. Sucrose intake and fasting glucose levels in 5-HT 1A and 5-HT 1B receptor mutant mice. Physiol Behav. 2008;93(4):659-665.

45. Olney JW, Ho OL. Brain damage in infant mice following oral intake of glutamate, aspartate or cysteine. Nature. 1970;227(5258):609-611.

46. Hill AJ. The psychology of food craving. Proc Nutr Soc. 2007:66(2):277285.

47. Warwick ZS, Weingarten HP. Dynamics of intake suppression after a preload: role of calories, volume, and macronutrients. Am J Physiol. 1994;266(4 Pt 2):R1314-R1318.

48. Bruinsma K, Taren DL. Chocolate: food or drug? Journal of the American Dietetic Association-Elsevier. 1999;99(10):1249-1256.

49. Stice E, Spoor S, Bohon C, et al. Relation of reward from food intake 
and anticipated food intake to obesity: a functional magnetic resonance imaging study. J Abnorm Psychol. 2008;117:924-935.

50. O'Doherty JP, Deichmann R, Critchley HD, et al. Neural responses during anticipation of a primary taste reward. Neuron. 2002;33(5):815-826.

51. Steven J St John, John D Boughter. Orosensory Responsiveness to and Preference for Hydroxide-Containing Salts in Mice. Chem Senses. 2009;34(6):487-498.

52. Herminghuysen D, Plaisance K, Pace R, et al. Prolonged stimulation of corticosterone secretion by corticotropin-releasing hormone in rats exhibiting high preference for dietary fat. Nutritional Neurosci. 1998;1(3):251-254.

53. Pomonis JJ. Sucrose consumption increases naloxone-induced c-Fos immunoreactivity in limbic forebrain. Am J Physiol. 2000; 278(3):R712R719.

54. Eric Stice, Sonja Yokum, Kenneth Blum, et al. Weight gain is associated with reduced striatal response to palatable food. $J$ Neurosci. 2010;30:13105-13109.

55. Olsen CM. Natural rewards, neuroplasticity, and non-drug addictions. Neuropharmacology. 2011;61:1109-1122.

56. Pepino MY, Mennella JA. Habituation to the pleasure elicited by sweetness in lean and obese women. Appetite. 2012;58(3):800-805.

57. Wang GJ, Volkow ND, Logan J, et al. Brain dopamine and obesity. Lancet. 2001;357(9253):354-357

58. Tordoff MG, Reed DR, Shao H. Calcium taste preferences: Genetic analysis and genome screen of C57BL/6 x PWK/PhJ hybrid mice. Genes brain behav. 2008;7(6): 618-628.

59. Van der Laan, Mark J Rose, Sherri. Targeted learning: causal inference for observational and experimental data. Springer Science \& Business Media; 2011

60. Portella AK, Kajantie E, Hovi P, et al. Effects of in utero conditions on adult feeding preferences. Journal of Developmental Origins of Health and Disease. 2012;3(3):140-152.

61. Di Marzo V, Matias. Endocannabinoid control of food intake and energy balance. Nat Neurosci. 2005;8(5):585-589.

62. Bellochio, Pauline Lafenêtre, Astrid Cannich et al. Bimodal control of stimulated food intake by the endocannabinoid system. Neuroscience. 2010;13:281-283.

63. Di Marzo V. Endocannabinoids: An appetite for fat. Proc Natl Acad Sci U S A. 2011;108(31):12567-12568.

64. Olszewski PK, Fredriksson R, Olszewska AM, et al. Hypothalamic FTO is associated with the regulation of energy intake not feeding reward. BMC neuroscience. 2009;10(1):12.

65. Simchen U, Koebnick C, Hoyer S, et al. Odour and taste sensitivity is associated with body weight and extent of misreporting of body weight. Eur J Clin Nutr. 2006;60(6):698-705.

66. Bartoshuk LM, Duffy VB, Hayes JE, et al. Psychophysics of sweet and fat perception in obesity: problems, solutions and new perspectives. Phil Trans Soc B. 2006;361(1471):1137-1148.

67. He K, Zhao L, Daviglus ML, et al. Association of Monosodium Glutamate Intake with overweight in Chinese Adults: The INTERMAP Study. Obesity. 2008;16( 8):1875-1880.
68. Beyreuther K, Biesalski HK, Fernstrom JD, et al. Consensus meeting: monosodium glutamate-an update. Eur J Clin Nut. 2007;61(3):304-313.

69. Olney JW. Brain lesions, obesity, and other disturbances in mice treated with monosodium glutamate. Science. 1969; 164(3880):719-721.

70. Zhang Y, Proenca R, Maffei M, et al. Positional cloning of the mouse obese gene and its human homologue. Nature. 1995;374(6521):452-532.

71. Monno A, Vezzani A, Bastone A, et al. Extracellular glutamate levels in the hypothalamus and hippocampus of rats after acute or chronic oral intake of monosodium glutamate. Neuroscience Letters. 1995;193(1):4548 .

72. Conlin PR. Eat your fruits and vegetables but hold the salt. Circulation 2007;116(14):1530-1531

73. Brown IJ, Tzoulaki I, Candeias V, et al. Salt intakes around the world implications for public health. Int J Epidemiol. 2009;38(3):791-813.

74. World Health Organization; 2013.

75. Avendano FP. Application of sensory evaluation in the food industry. Course Completion Work. Food Engineering, Institute of Food Science and Technology, Federal University of Rio Grande do Sul, Porto Alegre 2007.

76. Delwiche JF. The impact of perceptual interactions on perceived flavor Food Quality and Preference. 2004;15:137-146.

77. Di Marzo V, Goparaju SK, Wang L, et al. Leptin-regulated endocannabinoids are involved in maintaining food intake. Nature. 2001;410(6830):822-825.

78. Havel PJ. Control of energy homeostasis and insulin action by adipocyte hormones: leptin, acylation stimulating protein, and adiponectin. Curr Opin Lipidol. 2002;13(1):51-59.

79. Institute Of Food Technologists. Sensory evaluation guide for testing food and beverage products. Food Technology. 1981;35(11):50-59.

80. Mattes RD, Popkin BM. Nonnutritive sweetener consumption in humans: effects on appetite and food intake and their putative mechanisms. Am J Clin Nutr. 2009;89(1):1-14.

81. Mennella J, Jagnow C, Beauchamp G. Prenatal and postnatal flavor learning by human infants. Pediatrics. 2001;107(6):E88.

82. Morgane PJ, Mokler DJ, Galler JR. Effects of prenatal protein malnutrition on the hippocampal formation. Neuroscience \& Biobehavioral Reviews. 2002;26(4)471-483

83. Rozin P, Vollmecke TA. Food likes and dislikes. Annu Rev Nutr. 1986;6:433-456.

84. Sclafani A, Springer D. Dietary obesity in adult rats: similarities to hypothalamic and human obesity syndromes. Physiol Behav. 1976;17(3):461-471.

85. Speakman J, Hambly C, Mitchell S, et al. The contribution of the animal model to the study of obesity. Lab Anim. 2008;42(4):413-432.

86. Wolf ME. The Bermuda Triangle of cocaine-induced neuroadaptations. Trends Neurosci. 2010;33(9):391-398.

87. Yamamoto T. Central mechanisms of roles of taste in reward and eating Akta Phisiologika Hungarika; 2008

88. Zald DH. Orbitofrontal Cortex Contributions to Food Selection and Decision Making. Ann Behav med. 2009;38(Suppl 1):S18-S24. 